\title{
Distinguished Preduals of Spaces of Holomorphic Functions
}

\author{
CHRISTOPHER BOYD
}

ABSTRACT. For $U$ open in a locally convex space $E$ it is shown in [13] that there is a complete locally convex space $G(U)$ such that $\mathrm{G}(U)_{i}^{\prime}=\left(\mathscr{H} \mathcal{L}(U), \tau_{\delta}\right)$. We will show that when $U$ is balanced there is an $\mathscr{S}$-absolute decomposition for $G(U)$ in terms of the preduals of the spaces of homogeneous polynomials. For $U$ balanced open in a Fréchet space we investigate neccessary and sufficient conditions for $\left(\mathscr{C}(U), \tau_{\delta}\right)$ to be equal to $G(U)_{b}^{\prime}$.

\section{INTRODUCTION}

Let $U$ be an open subset of a locally convex space $E$ over $\mathbb{C}$ and let $\mathscr{X}(U)$ be the space of holomorphic functions from $U$ into $C$. We will denote by $\tau_{o}$ the compact-open topology on $\mathscr{H}(U)$. A semi-norm $p$ on $\mathscr{H}(U)$ is said to be ported by the compact subset $K$ of $U$ if for each open $V, K \subset V \subseteq U$, there is $C_{V}>0$ such that

$$
p(f) \leq C_{V}\|f\|_{V}
$$

for all $f \in \mathscr{H}(U)$. The $\tau_{\omega}$-topology on $\mathscr{H}(U)$ is the topology generated by all semi-norms ported by compact subsets of $U$.

If $K$ is a compact subset of $E$ we denote by $\mathscr{Z}(K)$ the space of holomorphic germs on $K$. The $\tau_{o}$ and $\tau_{\omega}$ topologies are defined by

1991 Mathematics Subject Classification: 46 G20.

Editorial Complutense. Madrid, 1993. 


$$
\left(\mathscr{H}(K), \tau_{o}\right)=\lim _{K \vec{C} U}\left(\mathscr{H}(U), \tau_{o}\right)
$$

and

$$
\left(\mathscr{K}(K), \tau_{\omega}\right)=\lim _{\overrightarrow{K \subset} U}\left(\mathscr{H}(U), \tau_{\omega}\right)
$$

Let $U$ be an open subset of a locally convex space $E$. We say that a seminorm $p$ on $\mathscr{X}(U)$ is $\tau_{\dot{b}}$-continuous, if for each countable increasing open cover $\left\{U_{n}\right\}_{n}$ of $U$ there is an integer $n_{o}$ and $C>0$ such that

$$
p(f) \leq C\|f\|_{n_{n_{0}}}
$$

for every $f$ in $\mathscr{H}(U)$. The $\tau_{\delta}$-topology on $\mathscr{H}(U)$ is the topology generated by all $\tau_{\delta}$ continuous semi-norms.

In [10], Mazet shows that there is a locally convex space $G(U)$ and a holomorphic map $\delta_{U}$ from $U$ into $G(U)$ with the following universal property: Given any complete locally convex space $F$ and $f \in \mathscr{L}(U, F)$ there is a unique $T_{f} \in \mathscr{Z}(G(U), F)$ such that $f=T_{f} \circ \delta_{U}$. In particular if we take $F=\mathbb{C}$, we see that $G(U)$ is a predual of $\mathscr{H}(U)$. Mujica and Nachbin [13] give a new proof of this theorem and show that $G(U)$ is also a topological predual and the inductive dual of $G(U), G(U)_{i}^{r}$, is equal to $\left(\mathscr{K}(U), \tau_{\delta}\right)$. In $\S 2$ we show that the spaces $\left(P\left({ }^{n} E\right), \tau_{\omega}\right)$ and $\left(\mathscr{H}(K), \tau_{\omega}\right)$ also have topological preduals which we denote by $Q\left({ }^{n} E\right)$ and $G(K)$ respectively. We show that the spaces $\left\{Q\left({ }^{n} E\right)\right\}_{n}$ are an $\mathscr{S}$-absolute decomposition for $G(U)$ when $U$ is balanced and therefore many of the topological properties of $G(U)$ can be obtained from the topological properties of $Q\left({ }^{n} E\right)$.

In the final section we assume $U$ is a balanced open subset of a Fréchet space. We show that we can construct $\left(\mathscr{X}(U), \tau_{o}\right)_{b}^{\prime}$ from the $G(K)$ 's, and use this result to show that $G(U)_{b}^{\prime}=\left(\mathscr{Z}(U), \tau_{\dot{\delta}}\right)$ if and only $\left(\mathscr{H}(U), \tau_{\delta}\right)$ is the bidual of ( $\left.\mathscr{H}(U), \tau_{o}\right)$.

If $E$ is a locally convex space and $n$ a positive integer, $\underset{n, n}{\hat{\otimes}} E$ will denote the $n$-fold tensor product of $E$ with itself completed with respect to and endowed with the $\pi$ or projective topology. We denote by $\underset{s, n, \pi}{\widehat{\otimes}} E$ the completion of the subspace generated by the symmetric tensors. 
We refer the reader to [6] for further reading on infinite dimensional holomorphy and to [9] for further reading on locally convex spaces.

\section{PREDUALS OF HOMOGENEOUS POLYNOMIALS AND SPACES OF GERMS}

Just as the space of holomorphic functions on each open subset of a locally convex space $E$ has a predual, the space of $n$-homogeneous polynomials on $E$, for each integer $n$, and the space of holomorphic germs on each compact subset $K$ of $E$ will also have a predual. In fact, for $n$-homogeneous polynomials, by taking $Q\left({ }^{n} E\right)$ to be the space of all linear forms on $P\left({ }^{n} E\right)$ which when restricted to each locally bounded set is $\tau_{\sigma^{-}}$ continuous, the proof of Theorem 2.1 of [13] is easily adapted to show the following:

Proposition 1. Let $E$ be a locally convex space, then for each positive integer $n$, there is a complete locally convex space $Q\left({ }^{n} E\right)$ and an $n$-homogeneous polynomial $\delta_{n} \in P\left({ }^{n} E, Q\left({ }^{n} E\right)\right)$ with the property that given any complete locally convex space $F$ and any $P \in P\left({ }^{n} E, F\right)$ there is a unique $L_{P} \in \mathscr{L}\left(Q\left({ }^{n} E\right), F\right)$ such that $P=L_{P} \circ \delta_{n}$.

This result has previously been proved by Mujica, [12], for Banach spaces and Ryan in [14] with $Q\left({ }^{n} E\right)$ replaced by $\hat{\otimes} E$. By the uniqueness of $L_{P}$ it will follow that $Q\left({ }^{n} E\right)$ is topologically isomorphic to $\underset{s, n, \pi}{\widehat{\otimes}} E$.

Let us define $G(K)$ to be the space of linear maps from $\mathscr{X}(K)$ to $\mathbb{C}$ which are $\tau_{\mathrm{o}}$-continuous on each set of holomorphic germs which are defined and uniformly bounded on some neighbourhood of $K$. Applying Theorem 1.1 of $[13]$ to the inductive limit $\left(\mathscr{K}(K), \tau_{\omega}\right)=\lim _{\overrightarrow{K \subset} Y} \mathscr{C H}^{\infty}\left(V, \| H_{v}\right)$ we get:

Proposition 2. Let $K$ be a compact subset of a locally convex space $E$, then $G(K)_{i}^{\prime}=\left(\mathscr{H}(K), \tau_{\omega}\right)$.

This result had been previously proved by Mujica [11] for $E$ a Fréchet space. In [11], Mujica points out that in this special case $G(K)=(\mathscr{H}(K)$, $\left.\tau_{o}\right)_{b}^{\prime}$. 
The concept of $\mathscr{S}$-absolute decomposition ([5]) allows us to obtain topological properties of $(\mathscr{Z}(U), \tau)$ from the corresponding properties of $\left(P\left({ }^{n} E\right), \tau\right), \tau=\tau_{o}, \tau_{\omega}$ or $\tau_{\delta}$ and $U$ balanced. To show that $\left\{Q\left({ }^{n} E\right)\right\}_{n}$ is an

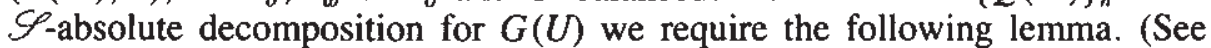
Proposition 3.15 of [6] for a related result.) We let $\mathscr{S}=\left\{\left(\alpha_{n}\right) \in \mathbb{C}^{\mathbb{N}}\right.$ : $\left.\lim \sup _{n \rightarrow \infty}\left|a_{n}\right| \frac{1}{n} \leq 1\right\}$.

Lemma 3. Let $U$ a balanced open subset of a locally convex space $E$, $\left(\alpha_{n}\right)_{n} \in \mathscr{S}$ and $\left\{f_{\beta}\right\}_{\beta}$ be a family of functions in $\mathscr{Z}(U)$ uniformly bounded on some neighbourhood of a compact balanced set $K$. Then there is an $M>0$ such that

$$
\sum_{n=0}^{\infty}\left|\alpha_{n}\right|\left\|\frac{\hat{d}^{n} f_{\beta}(0)}{n !}\right\|_{v} \leq M
$$

for every $\beta$ and some neighbourhood $V$ of $K$.

For $U$ open in any locally convex space $E$ it can be shown that, for each $n$, the map $\bar{\phi}: Q\left({ }^{n} E\right) \rightarrow G(U)$ defined by $\tilde{\phi}(f)=\phi\left(\frac{\hat{d}^{n} f(0)}{n !}\right)$ for $\phi \in Q\left({ }^{n} E\right), f \in \mathscr{H}(U)$ identifies $Q\left({ }^{n} E\right)$ with a closed subspace of $G(U)$.

Proposition 4. Let $U$ be a balanced open subset of a locally convex space $E$; then $\left\{Q\left({ }^{n} E\right)\right\}_{n}$ is an $\mathscr{S}$-absolute decomposition for $G(U)$.

Proof. Let $B=\left\{f_{\beta}\right\}$ be a family of locally bounded function in $\mathscr{H}(U)$. Recall that the topology on $G(U)$ is the topology of uniform convergence on locally bounded subsets of $\mathscr{H}(U)$. As

$$
\left(1,2^{2}, \ldots, n^{2}, \ldots\right) \in S^{P}
$$

it follows by Lemma 3 that for each $x$ we can choose a neighbourhood $V_{x}$ of $\Gamma_{x}$, the balanced hull of $\{x\}$, such that

$$
\sup _{\beta} \sum_{n=0}^{\infty} n^{2}\left\|\frac{\hat{d}^{n} f_{\beta}(0)}{n !}\right\|_{v_{x}}=M_{x}<\infty .
$$

Therefore for every $m$ and every $\beta$ we have, 


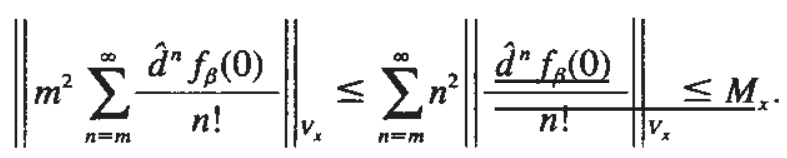

Thus the set

$$
\tilde{B}=\left\{m^{2} \sum_{n=m}^{\infty} \frac{\hat{d}^{n} f_{\beta}(0)}{n !}\right\}_{m, \beta}
$$

is locally bounded.

For $\phi \in G(U)$, let $\phi_{n}=\left.\phi\right|_{\left.P^{(}{ }^{*} E\right)}$. For each semi-norm $\alpha$ let $U_{\alpha}=\{x \in E$ : $\alpha(x) \leq 1\}$ and $B_{\alpha}^{n}=\left\{P \in P\left({ }^{n} E\right):\|P\|_{U_{a}} \leq 1\right\}$. Then $B_{a}^{n}$ is a locally bounded set of holomorphic functions on $\mathscr{X}(U)$ and $\phi_{n}\left|B_{a}^{n}=\phi\right| B_{a}^{n}$ is $\tau_{o}$-continuous and $\phi_{n} \in Q\left({ }^{n} E\right)$. Since $\phi$ is $\tau_{a}$-continuous on $\bar{B}$ and the Taylor series expansion of $f_{\beta}$ about 0 converges to $f_{\beta}$ in the $\tau_{o}$-topology we have that,

$$
\begin{aligned}
\left\|\phi-\sum_{k=0}^{m-1} \phi_{k}\right\| & =\sup _{\beta}\left|\left(\phi-\sum_{k=0}^{m-1} \phi_{k}\right)\left(f_{\beta}\right)\right| \\
& =\sup _{\beta}\left|\phi\left(\sum_{n=m}^{\infty} \frac{\hat{d}^{n} f_{\beta}(0)}{n !}\right)\right| \leq \frac{1}{m^{2}}\|\phi\|_{B} \rightarrow 0
\end{aligned}
$$

as $m \rightarrow \infty$. Thus $\phi=\sum_{n=0}^{\infty} \phi_{n}$ in $G(U)$.

As

$$
\left(\alpha_{1}, 2^{2} \alpha_{2}, \ldots, n^{2} \alpha_{n}, \ldots\right) \in \mathscr{S}
$$

for $\left(\alpha_{n}\right)_{n} \in \mathscr{S}$, it follows by Lemma 3 that for every $x \in U$, we can find $N_{x}>0$ such that

$$
\sup _{\beta} \sum_{n=0}^{\infty} n^{2} \alpha_{n}\left\|\frac{\hat{d}^{n} f_{\beta}(0)}{-\overline{n !}}\right\|_{w_{x}} \leq N_{x}
$$

for some neighbourhood $W_{x}$ of $\Gamma_{x}$. In particular the set

$$
B^{\prime}=\left\{n^{2} \alpha_{n} \frac{\hat{d}^{n} f_{\beta}(0)}{n !}\right\}_{n, \beta}
$$


is locally bounded. Let $\phi_{\eta} \rightarrow 0$ in $G(U)$. As every locally bounded subset of $P\left({ }^{n} E\right)$ is locally bounded in $\mathscr{Z}(U)$ and

$$
\left\|\left(\phi_{\eta}\right)_{n}\right\|_{\left\{\frac{\hat{a}^{n} f_{\beta}(0)}{n !}\right\}_{\beta}}=\sup _{\beta}\left|\phi_{\eta}\left(\frac{\hat{d}^{n} f_{\beta}(0)}{n !}\right)\right| \leq \frac{1}{n^{2} \alpha_{n}}\left\|\phi_{\eta}\right\|_{B^{\prime}},
$$

$\left(\phi_{\eta}\right)_{n} \rightarrow 0$ in $Q\left({ }^{n} E\right)$ for every $n$. This shows that $\left\{Q\left({ }^{n} E\right)\right\}_{n}$ is a Schauder decomposition for $G(U)$. For $\sum_{n=0}^{\infty} \phi_{n} \in G(U)$ and $\left(\alpha_{n}\right)_{n} \in \mathscr{S}$,

$$
\begin{aligned}
\left\|\sum_{n=k}^{\infty} \alpha_{n} \phi_{n}\right\|_{B} & \leq \sum_{n=k}^{\infty} \mid \alpha_{n}\left\|\phi_{n}\right\|_{B} \\
& =\sum_{n=k}^{\infty} \sup _{\beta}\left|\phi\left(\alpha_{n} \frac{\hat{d}^{n} f_{\beta}(0)}{n !}\right)\right| \\
& =\sum_{n=k}^{\infty} \frac{1}{n^{2}} \sup _{\beta}\left|\phi\left(n^{2} \alpha_{n} \frac{\hat{d}^{n} f_{\beta}(0)}{n !}\right)\right| \\
& \leq\|\phi\|_{B} \sum_{n=k}^{\infty} \frac{1}{n^{2}} .
\end{aligned}
$$

This shows that $\left\{Q\left({ }^{n} E\right)\right\}_{n}$ is an $\mathscr{S}$-decomposition for $G(U)$, and taking $k=0$, we see that the decomposition is $\mathscr{S}$-absolute.

In a way similar to that in which each $Q\left({ }^{n} E\right)$ can be identified with a closed subspace of $G(U)$ it can be shown that each $Q\left({ }^{n} E\right)$ can be identified with a closed subspace of $G(K)$ for $K$ a compact subset of $E$ and $E$ any locally convex space. By a modification of Proposition 4 we have:

Proposition 5. Let $K$ be a balanced compact subset of a locally convex space $E$, then $\left\{Q\left({ }^{n} E\right)\right\}_{n}$ is an $\mathscr{P}$-absolute decomposition for $G(K)$.

Corollary 6. If $E$ is a Fréchet space then $Q\left({ }^{n} E\right)=\left(P\left({ }^{n} E\right), \tau_{o}\right)_{b}^{\prime}$.

Proof. Both $\left\{Q\left({ }^{n} E\right)\right\}_{n}$ and $\left\{\left(P\left({ }^{n} E\right), \tau_{o}\right)_{b}^{\prime}\right\}_{n}$ are $\mathscr{P}_{\text {-absolute decomposi- }}$ tions for $G(K)=\left(\mathscr{H}(K), \tau_{o}\right)_{b}^{\prime}$. 


\section{DISTINGUISHED PREDUALS OF SPACES OF HOLOMORPHIC FUNCTIONS}

In this section we investigate conditions for $G(U)_{b}^{\prime}$ to be equal to $\left(\mathscr{H}(U), \tau_{\delta}\right)$. We begin by relating $\left(\mathscr{H}(U), \tau_{o}\right)_{b}^{\prime}$ to the $G(K)$ 's when $U$ is a balanced open subset of a Fréchet space. We denote by $R_{K, U}$ the map from $\mathscr{H}(U)$ to $\mathscr{H}(K)$ which assigns to each $f$ in $\mathscr{H}(U)$ its restriction to $K$.

Proposition 7. Let $U$ be a balanced open subset of a Fréchet space

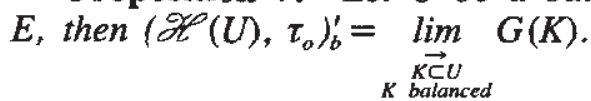

Proof. Since $\left(\mathscr{H}(U), \tau_{o}\right)$ and $\left(\mathscr{H}(K), \tau_{o}\right)$ are semi-Montel, we have that $\left(\mathscr{H}(U), \tau_{o}\right)_{b}^{\prime}=\left(\mathscr{H}(U), \tau_{o}\right)_{\tau}^{\prime}$ and $\left(\mathscr{H}(K), \tau_{o}\right)_{\tau}^{\prime}=\left(\mathscr{H}(K), \tau_{0}\right)_{b}^{\prime}=G(K)$, where $\tau$ denotes the Mackey topology. If $K$ is a balanced compact subset of $U$ then the Taylor series of $f$ about 0 converges to $f$ in $\left(\mathscr{H}(K), \tau_{0}\right)$. Since $\mathscr{U}(U)$ contains all polynomials, $R_{K, u}\left(\left(\mathscr{Z}(U), \tau_{\mathrm{o}}\right)\right)$ is dense in $\left(\mathscr{X}(K), \tau_{0}\right)$. Therefore the inductive limit

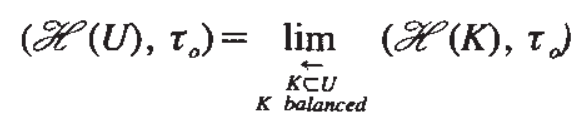

is reduced. Therefore by IV.4.4 of [15] we have that

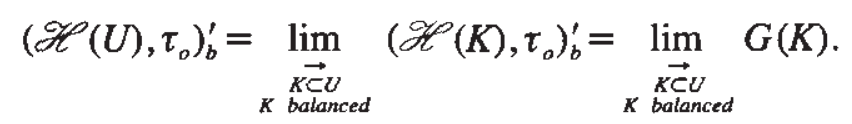

Lemma 8. Let $U$ be a balanced open subset of a Fréchet space $E$, then $G(U)_{b}^{\prime}=\left(\left(\mathscr{C P}(U), \tau_{o}\right)_{b}^{\prime}\right)_{b}^{\prime}$.

Proof. It follows by Grothendieck's Completeness Theorem, Theorem 3.11 .1 of [9], that $G(U)$ is the completion of $\left(\mathscr{H}(U), \tau_{o}\right)_{b}^{\prime}$. Therefore, $\left(\left(\mathscr{H}(U), \tau_{o}\right)_{b}^{\prime}\right)^{\prime}=G(U)^{\prime}=\mathscr{H}(U)$. To show that these two topologies agree 
it is sufficient to prove that each bounded subset of $G(U)$ is contained in the closure, in $G(U)$, of a bounded subset of $\left(\mathscr{C}(U), \tau_{o}\right)_{b}^{s}$. Let $B$ be a bounded subset of $G(U)$. Define $\vec{B}$ by

$$
\tilde{B}:=\left\{\sum_{n=0}^{m} \phi_{n}: \sum_{n=0}^{\infty} \phi_{n} \in B, m \in \mathbb{N}\right\},
$$

where $\phi_{n}=\left.\phi\right|_{\left.P_{(}{ }^{n} E\right)}$. By the proof of Proposition 3.13 of [6], $\tilde{B}$ is bounded and as $\phi_{n} \in Q\left({ }^{n} E\right)=\left(P\left({ }^{n} E\right), \tau_{o}\right)_{b}^{\prime}, \tilde{B} \subset\left(\mathscr{C}(U), \tau_{o}\right)_{b}^{\prime}$. Since $\sum_{n=0}^{m} \phi_{n} \rightarrow \phi$ on the locally bounded (and therefore $\tau_{o}$-bounded) subsets of $\mathscr{Z}(U), B$ is contained in the closure of $\tilde{B}$. This completes the proof.

The space $G(U)$ is not in general a Fréchet space when $U$ is an open subset of a Fréchet space $E$. However, our next result shows that $G(U)$ behaves very like a Fréchet space vis-a-vis necessary and sufficient conditions needed in order to show it is distinguished. We will denote by $\tau_{d}$ the topology on $\mathscr{H}(U)$ defined by $\left(\mathscr{H}(U), \tau_{d}\right)=G(U)_{b}^{\prime}$.

Theorem 9. Let $U$ be a balanced open subset of a Fréchet space E, then the following are equivalent:

(a) $G(U)_{b}^{\prime}=G(U)_{i}^{r}=\left(\mathscr{H}(U), \tau_{\delta}\right)$,

(b) $G(U)$ is distinguished $\left(G(U)_{b}^{\prime}\right.$ is barrelled),

(c) $G(U)_{b}^{\prime}$ is infrabarrelled,

(d) $G(U)_{b}^{\prime}$ is bornological.

Proof. Since $\left(\mathscr{H}(U), \tau_{\delta}\right)$ is barrelled and bornological we see that (a) will imply (b), (c) and (d).

It follows from Lemma 8 and the fact that $G(U)_{i}^{\prime}=\left(\mathscr{H}(U), \tau_{\delta}\right)$, that $\tau_{d}$ is a topology on $(U)$ which satisfies $\tau_{o} \leq \tau_{d} \leq \tau_{\delta}$. It now follows from Corollary 3 of [1] that (b) implies (a). Since $G(U)$ is barrelled, see Theorem 4.4 of [13], it follows by Theorem 3.6.1. of [9], that $G(U)_{b}^{\prime}$ is quasi-complete and so (b) is equivalent to (c). Finally, we note that (d) always implies(c).

Conditions (a) and (b) of the above Theorem are also true in the case where $U$ is a balanced open subset of a DF space where we replace Corollary 3 of [1] by Corollary 5 of [1]. 
From Lemma 8 and Theorem 9 we see that if $U$ a balanced open subset of a Fréchet space $E$, then $G(U)_{b}^{\prime}=\left(\mathscr{H}(U), \tau_{\delta}\right)_{b}^{\prime}$ if and only if the bidual of $\left(\mathscr{K}(U), \tau_{o}\right)$ is equal to $\left(\mathscr{H}(U), \tau_{\delta}\right)$. The question of when $\left.\left(\left(\mathscr{H}(U), \tau_{o}\right)\right)_{b}^{\prime}\right)_{b}^{\prime}$ is equal to $\left(\mathscr{H}(U), \tau_{\delta}\right)$, was investigated by Dineen and Isidro in [8]. There they proved the following Proposition.

Proposition 10. (Dineen-Isidro) Let $U$ be a balanced open subset of a locally convex space $E$, then $\left(\left(\mathscr{Z}(U), \tau_{o}\right)_{b}^{\prime}\right)_{b}^{\prime}=\left(\mathscr{K}(U), \tau_{\delta}\right)$ if and only if $\left(\mathscr{H}(U), \tau_{\delta}\right)$ has a basis of absolutely convex $\tau_{o}$-closed neighbourhoods of 0 .

Thus, in the case where $U$ is a balanced open subset of a Fréchet space $E$, we see that the sufficient condition given in [13] Theorem 1.1 for $G(U)_{b}^{\prime}$ to be equal to $\left(\mathscr{H}(U), \tau_{\delta}\right)$ is in fact also necessary.

As a complemented subspace of a distinguished space is distinguished, and $Q\left({ }^{n} E\right)$ is complemented in $G(U)$ for every integer $n$, we see that a necessary condition for $\tau_{d}$ to equal $\tau_{\delta}$ is that each $Q\left({ }^{n} E\right)$ is distinguished. This gives us a means of obtaining Fréchet spaces $E$ such that $G(U)_{b}^{\prime} \neq$ $G(U)_{i}^{\prime}$ for any balanced open subset $U$ of $E$. We begin with the observation that $Q\left({ }^{\prime} E\right)=E$, and therefore we have that $G(U)_{b}^{\prime} \neq\left(\mathscr{H}(U), \tau_{\delta}\right)$ for any balanced open subset of a non-distinguished Fréchet space.

Taskinen, [16], constructs a Fréchet-Montel space $F_{o}$, such that $Q\left({ }^{2} F_{o}\right)=F_{o} \widehat{\bigotimes}_{s, \pi} F_{o}$ is not distinguished. Thus we see that $G(U)_{b}^{\prime} \neq$ $\left(\mathscr{H}(U), \tau_{\delta}\right)$ for any balanced open subset $U$ of $F_{o}$. This also means that there are Fréchet-Montel spaces such that $G(U)$ is not distinguished.

To apply Theorem 9 and obtain examples of open subsets of Fréchet spaces where $G(U)_{b}^{\prime}=\left(\mathscr{Z}(U), \tau_{\delta}\right)$ is very difficult. This is because it is hard to show that an arbitrary locally convex space is distinguished. A necessary condition for $\tau_{d}$ to be equal to $\tau_{\delta}$ is that each $Q\left({ }^{n} E\right)$ is distinguished. When this holds we have the following Proposition showing that $\tau_{d}$ is finer than $\tau_{\omega}$.

Proposition 11. Let $E$ be a Fréchet space such that $Q\left({ }^{n} E\right)$ is distinguished for every integer $n$, then, for every balanced open set $U$ in $E, \tau_{d}$ is finer than $\tau_{\omega}$ on $\mathscr{H}(U)$. 
Proof. For every balanced compact subset $K$ of $E, G(K)$ is a Fréchet space, and it follows by Proposition 2 of [3], that $G(K)$ is distinguished. Therefore $G(K)_{i}^{\prime}=G(K)_{b}^{\prime}$, for every balanced compact set $K$ of $E$. Hence

$$
\left(\mathscr{H}(U), \tau_{\omega}\right)=\lim _{K \subset U}\left(\mathscr{H}(K), \tau_{\omega}\right)=\lim _{K \subset U} G(K)_{i}^{\prime}=\lim _{K \subset U} G(K)_{\forall}^{\prime}
$$

By the definition of projective limit, $\lim _{K \subset U} G(K)_{b}^{\prime}$. is weaker than

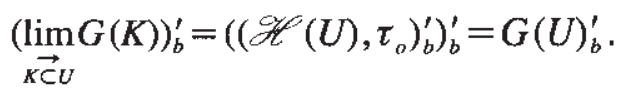

If $E$ is a Banach space, $Q\left({ }^{n} E\right)$ will also be a Banach space for every integer $n$. In particular, each $Q\left({ }^{n} E\right)$ is distinguished and therefore by Proposition 11 we have that $\tau_{\omega} \leq \tau_{d} \leq \tau_{\delta}$ on $\mathscr{H}(U)$ for every balanced open subset $U$ of $E$. Therefore if we know that $\tau_{\omega}=\tau_{\dot{\delta}}$ on $\mathscr{X}(U)$, we can conclude that $G(U)_{b}^{\prime}=\left(\mathscr{H}(U), \tau_{\delta}\right)$. For examples of Banach spaces and products of Banach spaces with nuclear spaces with this property we refer to $[4,2,7]$.

\section{Acknowledgements}

The results in this paper were proved in my Doctoral thesis submitted to the National University of Ireland in August 1992. I would like to thank my supervisor Professor S. Dineen for his assistance and encouragement and Professor J.Mujica for his comments.

\section{References}

[1] Ansemil, J. M. \& Ponte, S.: Topologies associated with the compact open topology on $\mathscr{H}(U)$, Proc. R.Ir. Acad., Vol.82A No. 1 (1982), 121128.

[2] Céré G.: Functionnelles analyiques sur certains espaces de Banach, Ann. Inst. Fourier, 21 2, (1971), 15-21.

[3] Díaz, J. C. \& Miñarro, A. M.: Distinguished Fréchet spaces and projective tensor products, DOGA Turk. J.Math., 14 (1990), 191208.

[4] DineEn, S.: Holomorphic function on $\left(C_{o}, X_{b}\right)$ Modules, Math. Ann., 196 (1972), 106-116. 
[5] DiNEEN, S.: Holomorphic function on locally convex topological vector spaces: I.Locally convex topologies on $\mathscr{L}(U)$. Ann. Inst. Fourier, 23 (1973), 19-54.

[6] Dineen, S.: Complex analysis on locally convex spaces, North-Holland Math. Studies, 57 (1981).

[7] DinEEN, S.: Holomorphic function and Banach-Nuclear decompositions of Fréchet spaces, preprint.

[8] DINEEN, S., \& ISIDRO, J.: On some topological properties of the algebra of holomorphic functions, Unpublished Manuscript.

[9] HonvÁTH, J.: Topological vector spaces and distributions, Vol.1, Addison-Wesley, Massachusetts (1966).

[10] MAZET, P.: Analytic sets in locally convex spaces, North-Holland Math. Studies, 89, 1989.

[11] Mú̉ICA, J.: A completeness criterion for inductive limits of Banach spaces, Functional Analysis, Holomorphy and Approximation Theoryll (Ed.G.I. Zapata), North-Holland Math. Studies, 68 (1984), 319-329.

[12] MÚJICA, J.: Linearization of bounded holomorphic mappings on Banach spaces, Trans Amer. Math. Soc., 324 (1991), 867-887.

[13] MÚJICA, J., \& NACHBIN, L.: Linearization of holomorphic mappings on locally convex spaces, J. Math. Pures Appl, 71, (1992), 543560.

[14] RyAN, R.: Applications of topological tensor products to infinite dimensional holomorphy, Thesis, Trinity College Dublin (1980).

[15] Schaefer, H. H.: Topological vector spaces, Springer-Verlag, Third Printing corrected (1971).

[16] TASKINEN, J.: Examples of non-distinguished Fréchet spaces, Ann. Acad. Sci. Fenn, Série A.I. 14 (1989), 75-88. 